\title{
El salto desde la Gestión de Información a la Gestión del Conocimiento
}

\author{
Javier Martínez Méndez \\ Grupo de Tecnologías de la Información \\ Departamento de Información y Documentación \\ Universidad de Murcia
}

\subsection{Resumen}

La última década presenta dos fenómenos paralelos y contradictorios en nuestro campo, la evolución de las Tecnologías de la Información ha consolidado la importancia del papel desempeñado por la gestión de los sistemas de información en las organizaciones y, al mismo tiempo, ha ocasionado serios problemas en los procesos de toma de decisiones por parte de los directivos. El origen reside en la diferencia existente entre información y conocimiento, conceptos que suelen confundirse con excesiva frecuencia propiciando fisuras en las organizaciones. Una adecuada gestión del conocimiento corporativo garantizará el éxito de las organizaciones dentro de un mercado tan competitivo como el actual. (Autor)

Palabras Clave: Gestión de Información. Gestión del Conocimiento. Tecnología de la Información. Sistemas de Información organizacionales.

\subsection{Abstract}

Last decade presents two phenomena, parallel and contradictory at the same time, in the field of Information Management. The development of Information Technology has consolidated its importance into the management of the organisations and, in turn, has brought out serious difficulties into the decision making processes. The origin of these problems resides in the existing differences between information and knowledge, terms that are mistaken with a lot of frequency. In order to guarantee the future of the organisation into the actual competitive market, it would be very interesting and necessary to provide it with an optimal knowledge management system inside it. (Author)

Keywords: Information Management. Knowledge Management. Information Technology. Organizational Information Systems 


\section{Introducción}

El hecho de que una organización pueda gestionar de una manera adecuada sus datos y que, al mismo tiempo, esa misma organización no pueda suministrar de una manera eficaz a sus directivos información necesaria para la toma de decisiones, puede parecer una extraña paradoja e incluso encontraríamos fácilmente quien lo llegara a negar al considerarlo, en cierta manera, absurdo o imposible. No obstante, esta situación resulta posible y está llegando a convertirse en una realidad acuciante para muchas organizaciones que se encuentran abocadas a pérdida de eficacia y competitividad dentro del mercado global en el que nos encontramos inmersos en la actualidad.

Realizando una primera aproximación a la identificación de las causas originarias de esta problemática, observamos que, en muchas situaciones se confunde información con conocimiento y que, confiados en la valía de sus sistemas de información, los directivos de las organizaciones no disponen de nociones suficientemente válidas sobre la realidad donde se encuentran inmersas las mismas y adoptan decisiones, a ciegas en algunos casos, que no siempre representan aciertos en la gestión. En otros casos, nos encontramos conque la toma de decisiones se ralentiza excesivamente por lo que esas organizaciones se convierten en organizaciones lentas que, a la larga, tienden a ser escasamente operativas a pesar de ser organizaciones suficientemente "bien informadas".

Procede pues, recapacitar sobre si el concepto de "gestión de información" resulta suficientemente amplio o si conviene replantearnos el mismo en la línea de profundizar aún más en lo que significa la gestión de los sistemas de información en las organizaciones, y los resultados que deben esperarse de los mismos para que éstas puedan desarrollar sus actividades en un clima de certidumbre a la hora de la toma de decisiones, de manera que se garantice su supervivencia. Este proceso de reflexión viene desarrollándose en la línea de establecer un marco de nivel superior el correspondiente a la "gestión del conocimiento", algo así como una nueva dimensión en el desarrollo de los sistemas de información organizacionales.

\section{La revolución informática}

Si por un momento nos detuviéramos en nuestro quehacer diario y meditáramos durante un breve espacio de tiempo sobre el ciclópeo impacto de la Informática en la totalidad de nuestras actividades cotidianas, nos encontraríamos contemplando uno de los procesos revolucionarios más arrolladores de la Historia de la Humanidad; Mercedes Caridad (1993) recoge esta cita de Yoneji Masuda: "al considerar la diferencia de máquina y sistemas en cada uno de los dos casos, observamos que la revolución de la energía motriz necesitó 57 años

Scire. $5: 1$ (en.-jun. 1999) 41-54. 
para desarrollar la máquina de Newcomen y para alcanzar las mil unidades construidas, y aún más de 35 años para que la máquina de James Watt se difundiera por las industrias modernas... En cambio, la difusión del ordenador tuvo lugar unas 4,6 veces más rápida que la de la máquina de vapor", postulados casi visionarios de Yoneji Matsuda, confiriéndole a la revolución informática una carácter de verdadera revolución industrial.

En esta misma línea, si nos circunscribimos al impacto de la Informática en el campo de la gestión de información, y si el mismo pudiera ser cuantificable, aumentaría el factor medio de incidencia en otros campos de la actividad en proporción exponencial. El grupo de trabajo de la UE, coordinado por el excomisario europeo Martin Bangemann (Europa y la Sociedad Global de la Información, 1999), recoge en sus exposiciones iniciales: "En todo el mundo, las tecnologías de la información y las comunicaciones están generando una nueva revolución industrial que ya puede considerarse tan importante y profunda como sus predecesoras. Es una revolución basada en la información, la cual es en sí misma expresión del conocimiento humano. Hoy en día, el progreso tecnológico nos permite procesar, almacenar, recuperar y comunicar información en cualquiera de sus formas (oral, escrita o visual), con independencia de la distancia, el tiempo y el volumen. Esta revolución dota a la inteligencia humana de nuevas e ingentes capacidades, y constituye un recurso que altera el modo en que trabajamos y convivimos".

Tanto en este informe como en la larga serie de recomendaciones y líneas de acción promovidas desde los distintos Estados y adoptadas de forma rápida por todo tipo de organizaciones se recoge la necesidad de configurar redes de comunicación de datos, de promoción de nuevos servicios y nuevos productos y del establecimiento de un nuevo mercado. En este nuevo entorno, las organizaciones reciben, gestionan y producen cada vez mayor información, y los sistemas de información clásicos comienzan a presentar una serie de carencias a la hora de satisfacer con la suficiente prontitud las demandas de los miembros de las mismas. En cierto modo, cabría pensar que estos sistemas, al aumentar de tamaño pierden elasticidad y corren el peligro de fragmentarse y de provocar fisuras en las organizaciones, incluso en aquellas que, a primera vista, parecen unos entes sólidos e inquebrantables. De forma similar, Tom Wilson (Wilson, 1999) expone que "los sistemas actualmente diseñados se evidencian como poco flexibles a la hora de responder a una necesidad de información perentoria, como las que surgen en el desarrollo de reuniones de trabajo entre los ejecutivos de una organización..."; y si en esa reunión se ha de adoptar alguna decisión trascendental para la misma, los sistemas de información introducen factores de incertidumbre adicionales que pueden ocasionar grandes pérdidas en el futuro.

Scire. $5: 1$ (en.-jun. 1999) 41-54. 

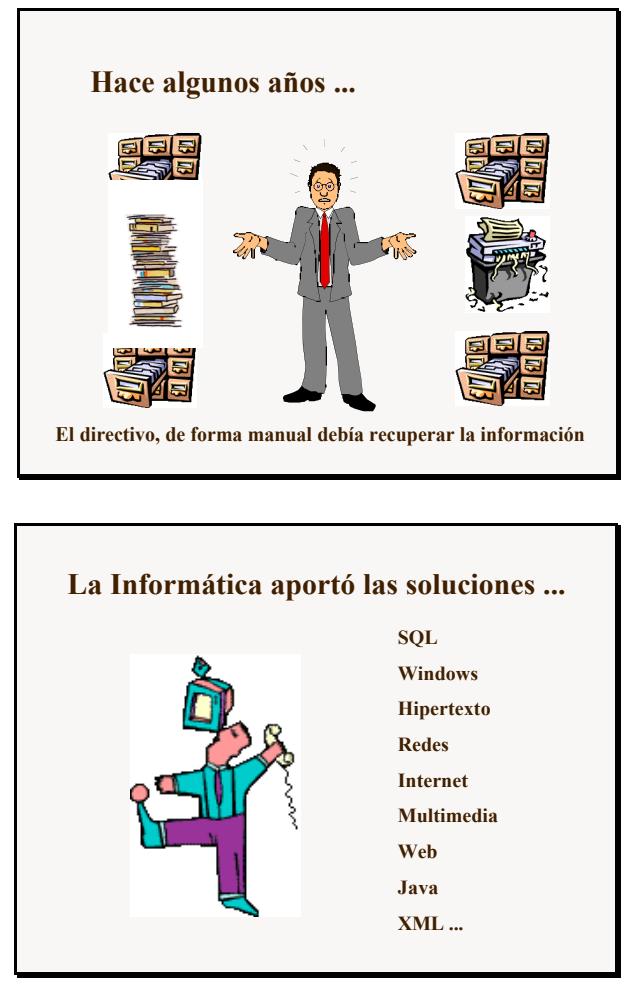

Con el tiempo, resulta que, en el fondo ...
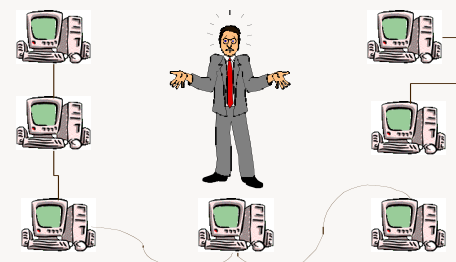

El directivo sigue desconociendo dónde acudir para recuperar la información: siguen los viejos problemas

Fig.1 ¿Por qué la Informática no resuelve la problemática?

Scire. $5: 1$ (en.-jun. 1999) 41-54. 


\section{3. ¿Qué ocurre?}

La concepción, por desgracia bastante extendida, de que la Informática resolvería los problemas de gestión de información, ha resultado ser equívoca, en tanto que, si bien en una primera instancia ha ayudado en ese proceso y ha generado grandes beneficios en las organizaciones, poco a poco se ha ido reproduciendo el problema cotidiano de ignorancia de la localización de los recursos disponibles y, lo que resulta aún peor, el desconocimiento de su propia existencia.

La diagnosis de este fenómeno nos introduce de nuevo en nuestra paradoja: el avance espectacular de la tecnología ha permitido la consolidación y desarrollo de los sistemas de gestión de la información; aunque, a la vez, este mismo avance produce un aumento exponencial de los documentos a almacenar, lo cual nos arrastra a un desbordamiento cognoscitivo.

De hecho, tanto en el lenguaje escrito (textos informativos, publicitarios e informes), como en el lenguaje oral, nos encontramos a menudo con afirmaciones de la clase de "disponemos de toda la información sobre...", "la base de datos más completa con la totalidad de las referencias a...", "en la red Internet encontraremos todo lo necesario para documentar los trabajo de clase...", o similares; enunciados todos ellos que si bien un verdadero profesional de la información valora como un simple acerbo propagandístico, estos textos pueden provocar que algunos responsables de organizaciones resulten inducidos a error y confíen decisiones a la disposición de ingentes cantidades de datos que no son capaces de gestionar, no digamos ya de asimilar, y por tanto, de extraer de ellos las consideraciones oportunas.

De hecho, la palabra "dato", no es siempre utilizada con acierto, en el DRAE (Real Academia Española, 1992) encontramos dos acepciones que nos sirven para ilustrar su verdadero significado: "antecedente necesario para llegar al conocimiento de una cosa" y "representación de una información de manera adecuada" (esta segunda acepción se realiza bajo un prisma informático"). Por consiguiente, la disposición de los datos resulta una condición necesaria para la posterior adquisición del conocimiento, pero no es una condición suficiente, nos falta algo. Verdaderamente, si de forma habitual, dedicáramos algún tiempo a la lectura del Diccionario de la Real Academia, percibiríamos que algunos errores tradicionalmente cometidos en muchas actividades, provienen de un uso incorrecto de la Lengua. En nuestro caso, nos encontramos con el término "información" (que frecuentemente se confunde con "dato" o con "conocimiento"), que significa "acción o efecto de informar o informarse", siendo "informar" la acción de "enterar, dar noticia de una cosa". Prosiguiendo con nuestra consulta al Diccionario, nos encontramos con el término "Conocimiento" que significa "acción y efecto de conocer", siendo el significado de "conocer" el siguiente: "

Scire. $5: 1$ (en.-jun. 1999) 41-54. 
averiguar por el ejercicio de las facultades intelectuales la naturaleza, cualidades y relaciones de las cosas". Realizando un pequeño proceso de abstracción y trasladándonos al entorno que nos preocupa, la gestión de los sistemas de información en las organizaciones representa una gestión de grandes colecciones de datos residentes en los grupos de ficheros informáticos gestionados por los sistemas gestores de bases de datos. Si a los procesos de gestión de estos sistemas de información le introducimos una serie de valores añadidos de carácter intelectual, como el estudio de las posibles sinergias entre los distintos sistemas gestionados en una misma organización y el aprovechamiento de las mismas, le estamos confiriendo una nueva dimensión, un valor añadido: el conocimiento.

Otra manera de presentar este mismo razonamiento nos la proporciona Neil Fleming (Fleming, 1999) que nos presenta el enunciado: "una colección de datos no es información, una colección de información no es conocimiento, una colección de conocimiento no es sabiduría y una colección de sabiduría no es necesariamente la verdad", redundando en la diferencia sustancialmente cognitiva que se establece entre la Gestión de la Información y la Gestión del Conocimiento.

\section{Solución al problema: la Gestión del Conocimiento}

Si no llegamos a comprender la diferencia entre datos e información, y si no distinguimos entre procesamiento primario de datos (sobre la información mis-

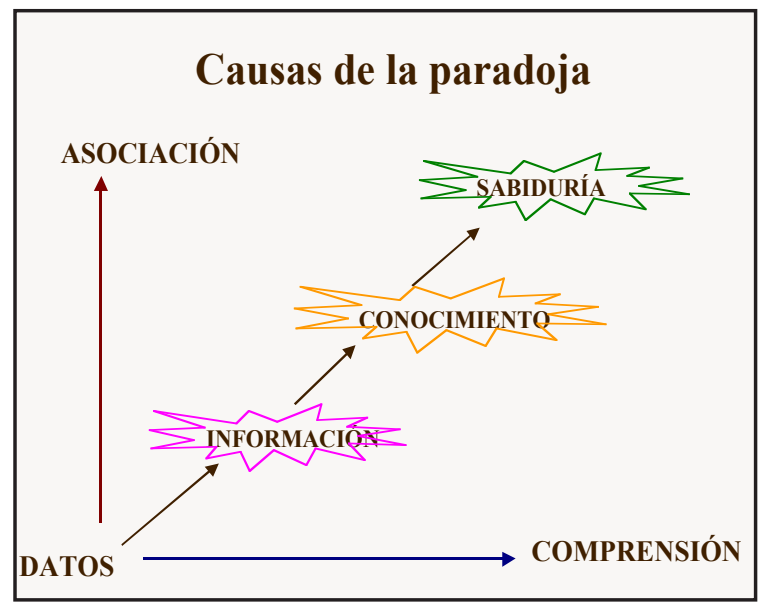

Fig. 2 Causas de la paradoja.

Scire. $5: 1$ (en.-jun. 1999) 41-54. 
ma) del procesamiento secundario de datos (los datos se usan para responder a las preguntas del proceso primario), crearemos costosos sistemas de información sin ningún beneficio para la organización. Una organización debe saber qué información es la que verdaderamente necesita. Si una organización no comprende la información que precisa, no sabrá qué información ha de almacenar y qué información necesitará con el paso del tiempo. Tampoco sabrá qué datos debe captar, ni cómo captarlos para que realmente le proporciones información, sobre la base de lo cual no se le plantea un futuro muy halagüeño a esta organización.

En una primera instancia, una vez percibida esta problemática, los gestores de estas organizaciones establecerían, en buena lógica, medidas conducentes a su resolución; y se verían tentados a adquirir nuevos equipos informáticos, con lo cual continuarían reiterándose en las causas originarias del problema, como nos dice Alfons Cornellá (Cornellá, 1999): "¿son las tecnologías de la información tan buenas? Según Paul Strassmann, conocido consultor norteamericano que ha estudiado con profundidad el impacto de las tecnologías de la información en las organizaciones, no hay una relación directa entre la inversión de las empresas en TI y el retorno que consiguen de esa inversión (puede verse al respecto, su web <http://www.strassmann.com>, en especial su artículo <http://www.strassmann.com/pubs/datamation0297>, en el que aparecen sus conocidos gráficos, que muestran la no existencia de tal relación). Strassmann dice que lo que hace rentable a las TI en una empresa no es el mero hecho de tenerlas, sino el cómo se utilizan"; de hecho, profundizando en las tesis de Paul Strassman, nos encontramos con la afirmación

obtener un resultado de la TI depende de cuan inteligentemente se gestionen. Y parte de esa inteligencia consiste en cambiar el chip mental

Es decir, tomando de nuevo comentarios de Alfons Cornellá: "hay que pasar a entender que la función de las TI es gestionar mejor la información, para convertirla en conocimiento, personal u organizacional... Pero, para conseguirlo, tenemos primero que conseguir entender que transferir información es muy poco útil, y que la clave esta en que los sistemas de información nos permitan intercambiar información: transaccionarla".

Joanne Miller (Miller, 1999) opina que:

A finales de los años 80 , se publicaron varios libros que describían una serie de procesos que superaban el concepto de la gestión de la información. En una de estas publicaciones, Cornelius Burk y Forest Horton describen cómo las corporaciones pueden identificar sus fuentes de información críticas. Estos autores introducen un nuevo pro eso que ellos denominan Information Resources Management, por medio del cual las organizaciones puedan distinguir la información de interés estratégico

Scire. $5: 1$ (en.-jun. 1999) 41-54. 
para la organización de la que no lo es tanto, identificando también aquellas fuentes de información que no le resulten productivas.

Posteriormente, ya en esta década, aparecen diversos trabajos donde se introducen nuevos términos como "inteligencia estratégica" o "capital intelectual", concepciones innovadoras que pretenden superar la tradicional de gestión de información.

Esta tendencia a acuñar un nuevo término responde a la verdadera necesidad que vienen planteando las organizaciones: disponer de un sistema de gestión del conocimiento plenamente operativo en su seno con la idea de economizar esfuerzos tanto humanos como materiales y de intentar aprovechar las sinergias positivas derivadas de una gestión transaccional de la información en la búsqueda de esas nociones fundamentales que aporten conocimiento sobre determinados temas a los gestores de las mismas. Introducimos en este punto el concepto de economía en los esfuerzos a realizar, concepto que se presenta como verdadera piedra angular de los sistemas de gestión del conocimiento. Debemos tener claro que cuando, en un momento dado, hay que acometer la realización de un determinado conjunto de procesos organizacionales, vamos a comprometer la utilización del conocimiento y se va a consumir una determinada cantidad de energía en recursos (humanos y materiales). Cuando, en otro momento determinado, vuelva a plantearse un proceso análogo pueden ocurrir dos situaciones:

- Reproducción del mismo consumo energético

- Minimización de dicho consumo, al disponer la organización de un sistema de gestión del conocimiento que permita reutilizar el conocimiento acumulado.

Así, cuando en un determinado departamento de nuestra organización se lleve a cabo algún desarrollo innovador, si en algún momento debe realizarse un proceso más o menos similar en alguna otra parte de la misma organización, el conocimiento individual adquirido por ese departamento redundará de forma positiva en la globalidad del conocimiento corporativo, redistribuyendo la posesión del conocimiento en la organización que puede ser utilizado para aportar mayor valor añadido a la información y por lo tanto, a los servicios prestados por la organización a sus clientes y a sus miembros.

Definir el concepto de gestión del conocimiento puede llegar a resultar un proceso más complicado de lo que podría parecer a simple vista, una vez determinadas sus ventajas y la necesidad de la misma. Realmente la gestión del conocimiento es más que un simple proceso apoyado en la tecnología, resulta algo más complejo. Otro de los problemas en su definición, lo encontramos en que existen dos tipos de conocimiento: intuitivo y explícito, resultando el primero de ellos difícil de discernir en algunos casos (y no digamos ya de procesar adecua- 
damente). Thomas Davenport (Davenport, 1996) la define como "el proceso sistemático de encontrar, seleccionar, organizar, destilar y presentar la información de una manera que mejore la comprensión de un área específica de interés para los miembros de una organización". Joanne Miller identifica una serie de procesos dentro de la gestión del conocimiento en una organización: "algunos de ellos incluyen la generación de nuevo conocimiento, el acceso al conocimiento sito en fuentes externas, la representación del conocimiento en documentos y en bases de datos, y el uso de este conocimiento en los procesos de toma de decisiones". Por tanto, engendrar conocimiento, reunirlo, compartirlo, distribuirlo y aplicarlo para la gestión de la organización, con acciones que sirvan para crear valor añadido, diferenciador y que genere una mayor eficacia en todas sus áreas es lo que se denomina gestión del conocimiento.

\section{5. ¿Cómo saltamos?}

Antes de proceder a este salto (pirueta en algunos casos), hay que tener presente dos aspectos importantes. En primer lugar, este paso a un nuevo contexto no debe llevarse a cabo de forma traumática, más si cabe porque la idea fundamental es aprovechar mejor los servicios que ya nos prestan nuestros actuales sistemas. En segundo lugar, este proceso de cambio va a afectar a toda la organización en su conjunto, no debe circunscribirse a áreas específicas porque en todos los niveles de la misma, en mayor o menor medida, se procesa ese conocimiento que tenemos que procurar gestionar de la mejor forma posible.

La forma más adecuada para comenzar a introducir en nuestra organización un sistema de gestión del conocimiento es por medio de una reingeniería de los procedimientos, proceso a través del cual se deben integrar los sistemas transaccionales implantados para la gestión de la información: sistemas de gestión documental; gestores de bases de datos; sistemas hipertexto abiertos y distribuidos (intranets, extranets); herramientas de búsqueda y recuperación de la información (motores y agentes); y tecnologías para la colaboración entre grupos de trabajo (mail y sistemas de groupware y workflow).

Sea cual sea el sistema que se implante, siempre habrá de reconocer, en primer lugar, los procedimientos por medio de los cuales los trabajadores desarrollan sus tareas y construir a partir de ahí las soluciones tecnológicas alrededor de los siguientes procesos:

- Producción de Información: El tratamiento de textos es la herramienta fundamental para la creación de información y el elemento principal de generación de conocimiento, llegando algunos a incluir prestaciones altamente sofisticadas. A su lado coexiste una amplia gama de herramientas de producción de información: software de autoedición, aplicaciones CAD, los

Scire. $5: 1$ (en.-jun. 1999) 41-54. 
navegadores de Internet, las hojas electrónicas o los sistemas de gestión de bases de datos, etc. Puestas todas estas herramientas al servicio del trabajador del conocimiento, generan nuevas salidas electrónicas de información que de forma aislada o conjunta con otros segmentos de información conforman un complejo producto basado en el conocimiento.

- Acceso y Distribución de la Información: La información externa e interna existe, por lo que la actuación se centra tanto en la forma de acceder a ella como en la manera de distribuirla. Para los trabajadores del conocimiento su principal preocupación será cómo obtener la información y cómo distribuirla adecuadamente. Uno de los aspectos más importantes relativos al acceso a la información es su formato, lo que produce la necesidad de compatibilizar distintos tipos de plataformas, de sistemas operativos, de gestores de bases de datos y de protocolos de comunicaciones. Para ello se dispone de un considerable conjunto de soluciones que permiten organizar recursos para la distribución y debate de la información, como son los sistemas groupware y de workflow que facilitan una mayor fluidez en dinámica en grupo de los procesos de trabajo.

- Entornos «push»: Los agentes nos proporcionan información en bloques, clasificando, midiendo y filtrando inmensas cantidades de información. Constan de un sistema de indexación de información, que facilita la rápida búsqueda y recuperación a un amplio número de usuarios. Los últimos desarrollos acomodan las preferencias de las búsquedas a las necesidades de los trabajadores, utilizando agentes inteligentes de búsqueda del conocimiento, diseñados para actuar en nombre de un usuario en la búsqueda: qué han de buscar, dónde y cuándo buscarla, cuándo entregar los resultados de la búsqueda y en qué formato y estructura presentarlos, acercando la información directamente a los usuarios cuando éstos la necesitan, más que cuando de forma activa quieran buscarla.

- El Mapa de la Información: Define los canales disponibles para su utilización por usuarios individuales o por toda la organización. Se configura como un esquema general que describe los mecanismos disponibles para el proceso de la información y para la formulación del conocimiento. Para que un sistema de estas características sea eficaz para el usuario, ha de ser fácilmente accesible, flexible e intuitivo. El Mapa de Información asegura que todos los usuarios conozcan lo que tienen a su disposición; contribuyendo a mejorar la agilidad en la comunicación interna en el seno de la organización o la facilidad para la toma de decisiones 
Con la integración de toda esta serie de tecnologías en el seno de una organización se obtienen una amplia serie de resultados que nos resume Rodríguez Rovira (Rodríguez Rovira, 1999):

- Mejora de la calidad en productos y servicios.

- Mejora de la atención a los clientes.

- Mejora de las relaciones con los proveedores.

- Creación de condiciones para mejorar el ambiente de trabajo.

- Mejora de la comunicación interpersonal.

- Estimulación de la participación de los trabajadores.

- Reducción del número de procesos de gestión/producción.

- Simplificación de los procesos de gestión/producción.

- Aumento de la eficiencia en el uso de los recursos.

- Diseño de nuevas y mejores herramientas para la gestión de la dirección

Para culminar con éxito este proceso de cambio, hacen falta una serie de elementos adicionales, como son:

- Un Modelo para el diseño del sistema de gestión del conocimiento

- El sistema en sí mismo

- El profesional o conjunto de profesionales encargados de esta tarea

Proceder a un estudio exhaustivo de los diversos modelos más aceptados para proceder al desarrollo de sistemas de gestión del conocimiento y de los sistemas implantados en la práctica que han resultado más adecuados, rebasa las pretensiones de este trabajo, que pretende centrarse en el tercer elemento: el trabajador. Para un estudio amplio tanto de modelos como de sistemas, se recomienda la consulta al sitio de Internet denominado "WWW Virtual Library on Knowledge Management" puesto en funcionamiento por Yogesh Malhotra (Malhotra, 1999a).

\section{El trabajador del conocimiento}

Hemos indicado anteriormente que en mayor o menor medida, todos los trabajadores de una organización se van a ver involucrados en este proceso de gestión del conocimiento. De hecho, Nonaka y Takeuchi (Nonaka y Takeuc, 1995) afirman que engloba "al menos a todos los miembros de una organización"; Joanne Miller advierte que "un sistema de gestión del conocimiento implica la creación de nuevos perfiles profesionales y de unidades organizacionales". Yogesh Malhotra (Malhotra, 1999b) expone que "en un nivel fundamental, el objetivo es lograr la sinergia entre las capacidades de las tecnologías de la infor-

Scire. $5: 1$ (en.-jun. 1999) 41-54. 
mación, y la creativa capacidad de la mente humana de los miembros de las organizaciones. Desde ahora, los trabajadores del conocimiento necesitan sentirse cómodos con la introducción de las nuevas tecnologías en sus entornos laborales, en tanto que su comprensión es totalmente necesaria para que puedan asimilar la idea general y tras percibirla en su globalidad, delegar las tareas rutinarias para concentrar todo su tiempo y esfuerzos a las actividades generadoras de valor añadido para la organización, que requieren de sus capacidades de creatividad e innovación".

Podríamos seguir presentado acepciones diferentes sobre este término, pero lo que nos interesa sobremanera es reflexionar si el actual profesional de la información puede llegar a convertirse en un verdadero trabajador del conocimiento. Parece lógico que así sea, superando toda la serie de reticencias iniciales tanto por parte de los gestores como por parte de los propios colectivos de profesionales. Joanne Miller afirma que la mayor parte de las organizaciones buscan fuera del colectivo de profesionales de la información a esos profesionales del conocimiento, tendencia que debe corregirse por medio de la formación y especialización de estos profesionales actuales y de los futuros en todas las técnicas y métodos relacionados con esta materia. Rodríguez Rovira apuesta por la conformación de equipos interdisciplinares donde participen los actuales profesionales de la información: "en algunas organizaciones se ha creado la figura del knowledge manager o gestor del conocimiento, como el especialista que tiene la responsabilidad de definir, impulsar, coordinar, dar coherencia y evaluar los procesos que configuran la $\mathrm{GdC}$. Esto comporta coordinar equipos de profesionales, dirigir procesos de actividades y, también, conocer y dirigir la implementación de las tecnologías y herramientas necesarias para la realización práctica. No hay una profesión concreta que cualifique para esta actividad, pero es evidente que los profesionales de la información/documentación reúnen unas condiciones inmejorables de partida si, además, están motivados en todos los aspectos de gestión y tecnología".

Coincidiendo con el último enunciado, ya nos manifestábamos en 1997 (Martínez Méndez, 1997) cuando afirmábamos en nuestra participación en el desarrollo del II Congreso IBERSID, organizado por la Universidad de Zaragoza: "que nosotros, los profesionales de la gestión información y de la documentación (llámense bibliotecarios, archiveros, documentalistas, gestores, infónomos...) debemos estar ahí, colaborando codo con codo con otros profesionales en el desarrollo de estos sistemas, y para ello debemos ir adaptando nuestros curricula incluyendo en ellos los contenidos formativos precisos para ejercer dicha actividad, olvidémonos ya de discusiones efímeras sobre proyectos abandonados hace más de un siglo y centrémonos en el estudio de la Ingeniería de la Información (o del Conocimiento)", postulado en el que nos reposicionamos

Scire. $5: 1$ (en.-jun. 1999) 41-54. 
(realmente nunca nos hemos movido), con el objeto de estar presentes en el próximo milenio, cada vez más cercano.

Y para concluir, queremos reincidir en que la gestión del conocimiento debe mejorar nuestros productos, y que dentro del entorno tan competitivo de hoy en día, la supervivencia de la organización viene determinada por la aceptación de los usuarios (que ya pueden elegir dentro de un mercado cada vez más amplio), por lo que el modelo de sistema de gestión del conocimiento que adoptemos como base para nuestros desarrollos debe ser, como nos recuerda Tom Wilson: "un modelo para el usuario, debe ser un modelo organizativo, no sólo un modelo de desarrollo de sistemas o un modelo de una sección individual de la organización, porque, en este caso, adolecerá de una perspectiva limitada de la organización y no asentará unas bases sólidas para el desarrollo del sistema de gestión del conocimiento".

\section{Bibliografía}

Caridad Sebastián, M. (1993). Fundamentos teóricos en Documentación Automatizada: Programa y Bibliografía. Barcelona, Murcia : PPU, Diego Marín Librero, 1993.

Cornellà Solans , A.(1999). La Ley de Moore, y ahora la Ley de Demi Moore. Barcelona : ESADE, 1998. URL=<http://www.extra-net.net/articulos/en980527.htm>.

Davenport, T.(1996). Knowledge Management [en línea]: Case Study, Knowledge Management at Hewlett-Packard, Early 1996". Austin: Universidad de Texas $\mathrm{URL}=<\mathrm{http}: / /$ www.bus.utexas.edu/kman/hpcase.htm>.

Europa y la Sociedad Global de la Información [en línea]: Recomendaciones al Consejo Europeo. (1999). Madrid: Midas-Net. URL=<http://www.info2000.csic.es/midasnet/docs/informebang/informebang.htm>.

Fleming, N.(1999). Coping with a Revolution [en línea]: Will the Internet Change Learning? Lincoln, NZ: University. URL=<http://www.lincoln.ac.nz/educ/learning.htm>.

Malhotra, Y.(1999a). WWW Virtual Library on Knowledge Management: Forums, Articles, Magazines, Events, Resources, Analyses and News. Brint : Fort Lauderdale. $\mathrm{URL}=\langle$ http://www.brint.com/km/ >.

Malhotra, Y. (1999b). Knowledge Management, Knowledge Organizations \& Knowledge Workers: A View from the Front Lines. Brint: Fort Lauderdale. <http://www.brint.com/ interview/maeil.htm/ >. Consulta: 19 de septiembre de 1999.

Martínez Méndez , F.J. (1997). Formación en sistemas de información electrónica y nuevos documentos. // SCIRE. 3: 2 (1997) 11-30.

Miller, J.(1999). Knowledge management: How organizations manage their resources. Berkeley: Universidad. URL $=<$ http://info.berkeley.edu/ jmiller/knowman.html $>$.

Nonaka, I. ; Takeuchi, H.(1995). The Knowledge Creating Company. Oxford: Oxford University Press, 1995.

Scire. $5: 1$ (en.-jun. 1999) 41-54. 
Real Academia Española. (1992). Diccionario de la lengua española. 21 a ed. Madrid : Espasa Calpe, 1994.

Rodríguez Rovira, J.M. (1999). Gestión del conocimiento. // El Profesional de la Información. $18: 3$ (marzo 1999).

Wilson, T.D. (1999). User Modelling : A global perspective // Anales de Documentación. 2(1999) 95-102. 